\title{
New concept for higher Robot position accuracy during thermography measurement to be implemented with the existing prototype automated thermography end-effector utilising an industrial robot and laser system
}

\author{
by S. Dutta and T. Schmidt
}

German Aerospace Center, Institute of Structures and Design, Center for Lightweight Production Technology, Am Technologiezentrum 4, 86159 Augsburg, Mobile: +49-173-5617742 (Dutta); +49-172-8343570 (Schmidt); Fax: +49821-5985883, somen.dutta@dlr.de, t.schmidt@dlr.de

\begin{abstract}
This paper introduces the development of prototype robot based thermography measuring system and a new concept for higher robot position accuracy for industrial applications. The developed thermography system is mounted at the end-effector of an industrial robot. Furthermore, a platform for offline programming is introduced, which enables the identification of measurement positions and the optimal settings for the robot's kinematic parameters. Many factors may affect the accuracy of created robot TCP positions and path planning. The new concept using thermography camera, which is also used for NDT, and a laser system helps to enhance the measurement position and orientation accuracy. The measuring position is projected with the laser system on the component and robot improves his orientation and position towards the given target through iteration process.
\end{abstract}

\section{Introduction}

The carbon fiber parts are taking more and more importance in the aeronautical field as aircraft makers are chasing the weight to comply with some of the environmental constraints. Due to certifications, composite parts, like metallic ones, need to be quality controlled. To reduce production cost, industry has a growing interest in the manufacturing process (see figure 1) automation with robotic systems. But this implies specific constraints: for example, the shape of a body panel without its stiffeners differs from CAD model however geometric parameters have to be verified. Furthermore modulated thermal wave during Lock-In Thermography diffuses laterally to the surrounding surfaces of measurement filed, which prevents continuous measure to the very next measurement field. Therefore for large components the thermography measurement position has to be distributed systematically to get homogenous intensity of measurement field. Autonomous navigation with NDT system on a large aircraft component (e.g. fuselage sections, wing structures or similar) is a major challenge for industry robot. Robot arms are versatile, flexible and widely used in manufacturing industries for various applications such as material handling, painting, welding and assembly. However, it is known that robots have better repeatability than absolute positioning accuracy. Normally robot repeatability accuracy is about 0.1 to $0.2 \mathrm{~mm}$ but the absolute positioning inaccuracy may be up to $20 \mathrm{~mm}$ or even more if not properly calibrated. The problem can be basically divided into positioning and path planning. Robot Tool-Center-Point (TCP) positions with the best accuracy depending on robot pose, position and their orientation. Many factors may affect the accuracy of created robot TCP positions.

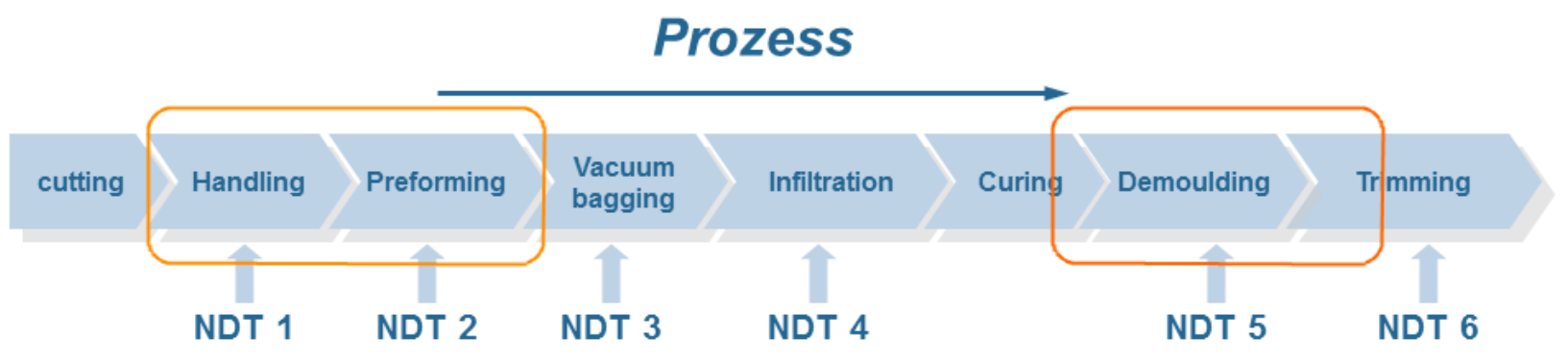

Figure 1: Schematic CFRP manufacturing process with inline NDT highlighted 
The new concept using thermography camera, which is also used for NDT, and a laser system mounted above the Robotic cell, helps to enhance the measurement position and orientation accuracy. The measurement position is projected with the laser system on the component, so that the thermography end-effector can detect the measurement position and robot improves his orientation and position towards the given target through iteration process.

\section{Description of current usage}

\subsection{Production integrated automated thermography}

During the manufacturing process it is desirable to examine parts to confirm they are defect free and fit for service without destroying the part. Large object measurements are always difficult and very expensive to automate. The main reason is that most large objects are bigger than the working envelope of measuring machines. Therefore DLR Augsburg with KUKA Systems has developed 3 different Robotic (see Figure 2) cells to be flexible enough and solve the automation purpose. The integration of a robot and a set of Halogen lamps and thermography camera offer a workbench for contactless nondestructive testing. In this system, robot calibration, camera calibration and measuring methodologies are the main processes in this study.
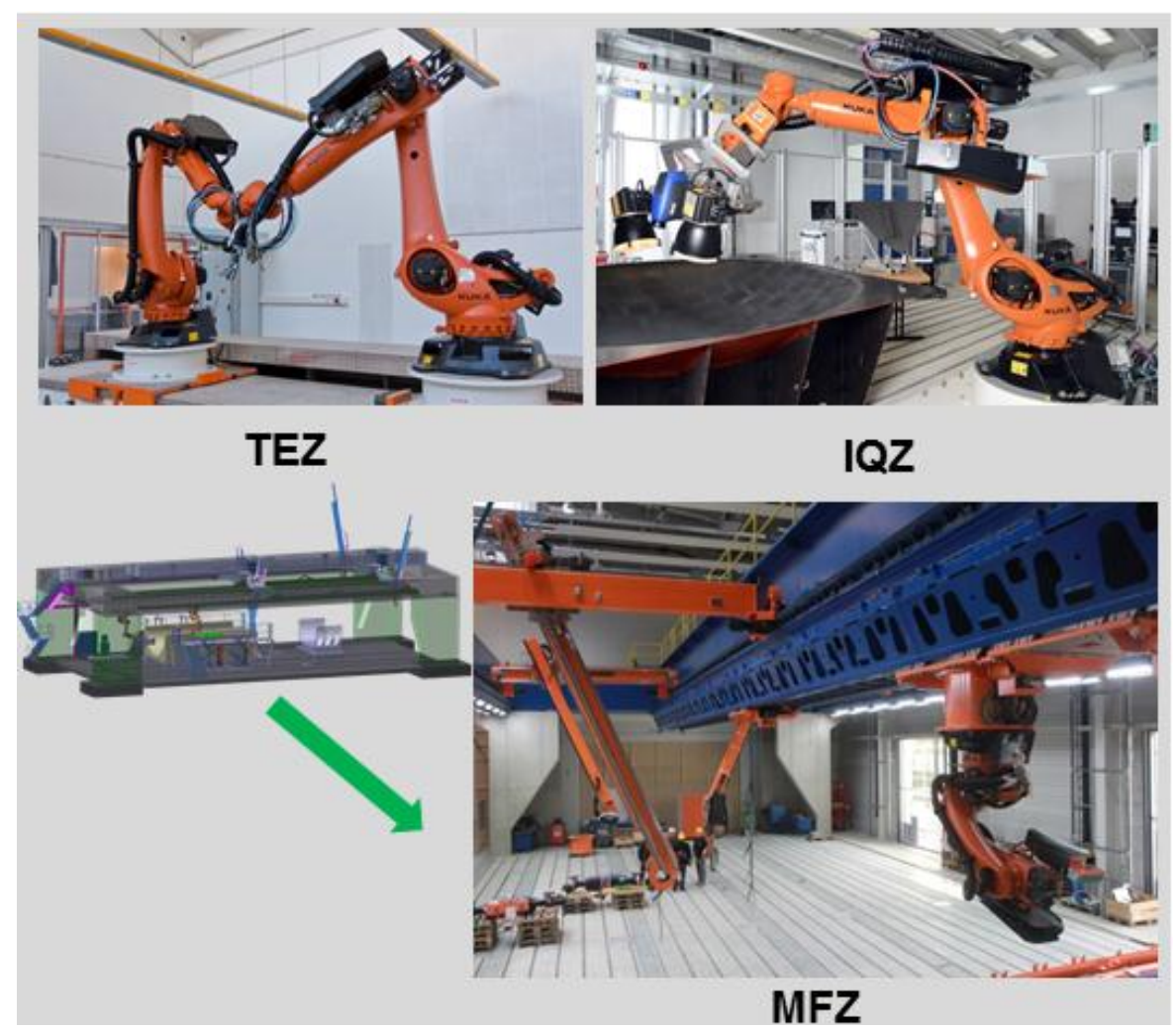

Figure 2: Three different Robot Cells at DLR

\subsection{Development and Integration of prototype thermography End-effectors}

The developed measurement system consists of two or more halogen lamps and one IR thermography camera, which is mounted at the end-effector of an industrial robot to ensure reliable defect detection. With the diverse adjustability of angles and positions of the lamps and IR camera, this prototype design is flexible to measure from longer as well as from shorter distances, 3D curved and difficult accessible measurement area. This makes the presented system well suited for the automated measurement of large tool parts. Furthermore, a platform for offline programming is introduced, which enables the identification of measurement positions and the optimal settings for the robot's kinematic parameters. 

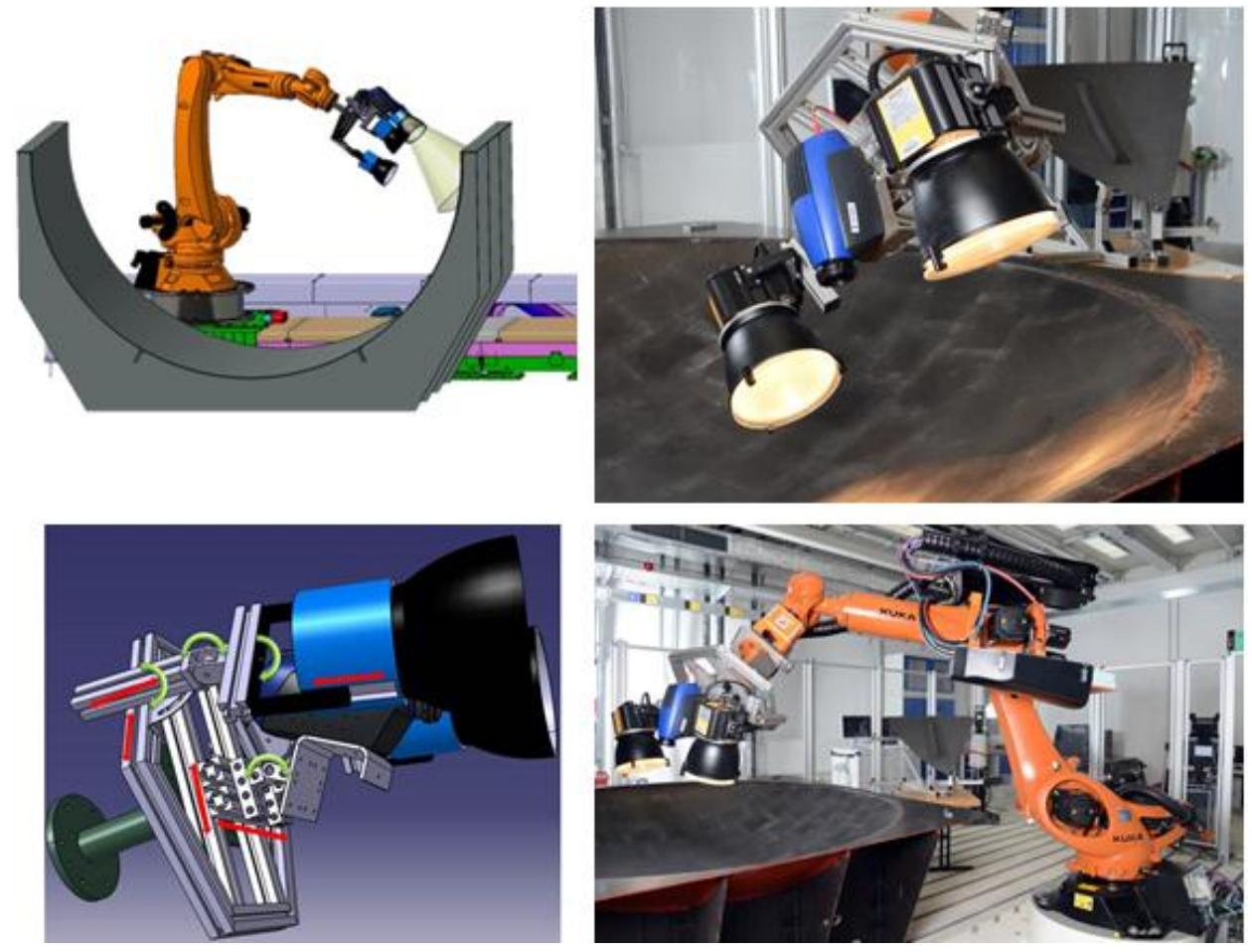

Figure 3: Robotic end-effector mounted on industrial robot

The proposed system and schemes are evaluated and verified experimentally. The experiment results have demonstrated the feasibility of implementation of prototype end-effectors and introduced the applicable measuring scope for the inline quality assurance. This is the first of its kind of prototype measuring systems and it evolved from earlier laboratory based systems. The project was focused on implementation and supervision of prototype systems and the development of measurement hardware. The paper [1 and 2] describes the design of the measuring system, presents an evaluated certainty of production integrated measurement system and outlines of test results obtained from the prototype system. Figure 3 represents the prototype thermography end-effector.

\section{Robotics and automation planning challenges}

Industrial robots are used in many applications that require moderate levels of accuracy and repeatability. These applications include pick and place, light assembly, cutting, spray painting and welding. Unfortunately industrial robots are designed to have a good repeatability but not a good accuracy. The poor accuracy is caused by geometric factors, such as geometric parameters, joints offset errors and TCP definition, as well as by nongeometric factors such as compliances, thermal effects gear, encoder resolution, gearboxes backlashes and kinematic errors. In Addition to that the thermography end-effector (due to its flexibility) setup has to be changed according to the measurement position. This means the loading and mass gravity of the End-effector changes according to setting and these needs to be adjusted in robot configuration. One of the contributions of this paper is an experimental verification of the robot positioning accuracy influenced by loading, mass gravity and temperature for existing robot cell at DLR. Each of the following robot characteristics accuracy, repeatability, and resolution depends upon many factors that include, but are not limited to, friction, temperature, loading, and manufacturing tolerances.

\subsection{Experimental Setup and measurement results}

A precondition for identifying the parameters is the measurement of the robot TCP. The experimental evaluation was performed using a single robot in IQZ robot cell (see Figure 2) and robot with external Axis in TEZ robot cell (see Figure 2) with a Leica laser tracer. In the Sensor based method the position error is measured by means of Laser to reflector position. This reflector was placed on robot flange (center Plane of the no. 6 Axis) and the Laser tracer was best fitted into robot coordinate system. The experiment was focused to find out influences of mass gravity and loading (by changing data in machine configuration) of axis 3 and Axis 6 . Several experiments were performed in 
order to evaluate the performance of the robot cells. In this experiment the robot movements were programmed for random 12 positions in the robot cell IQZ. The experimental verification contrasts the absolute position accuracy to the measured absolute position by Leica laser tracer. For examples Figure 4 and 5 represents the positions deviation influenced by mass gravity and loading of axis no. 6 .

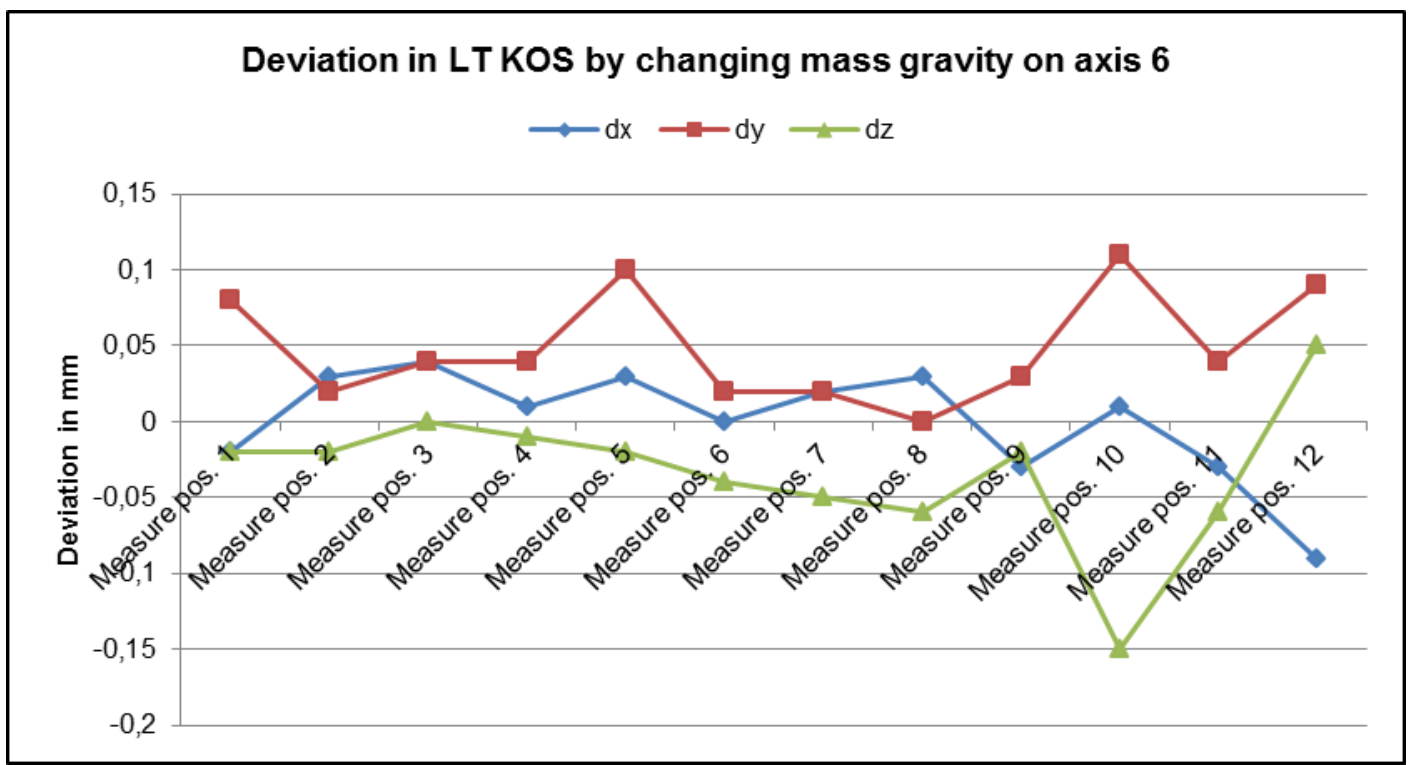

Figure 4: Position deviation influenced by mass gravity in IQZ robot cell

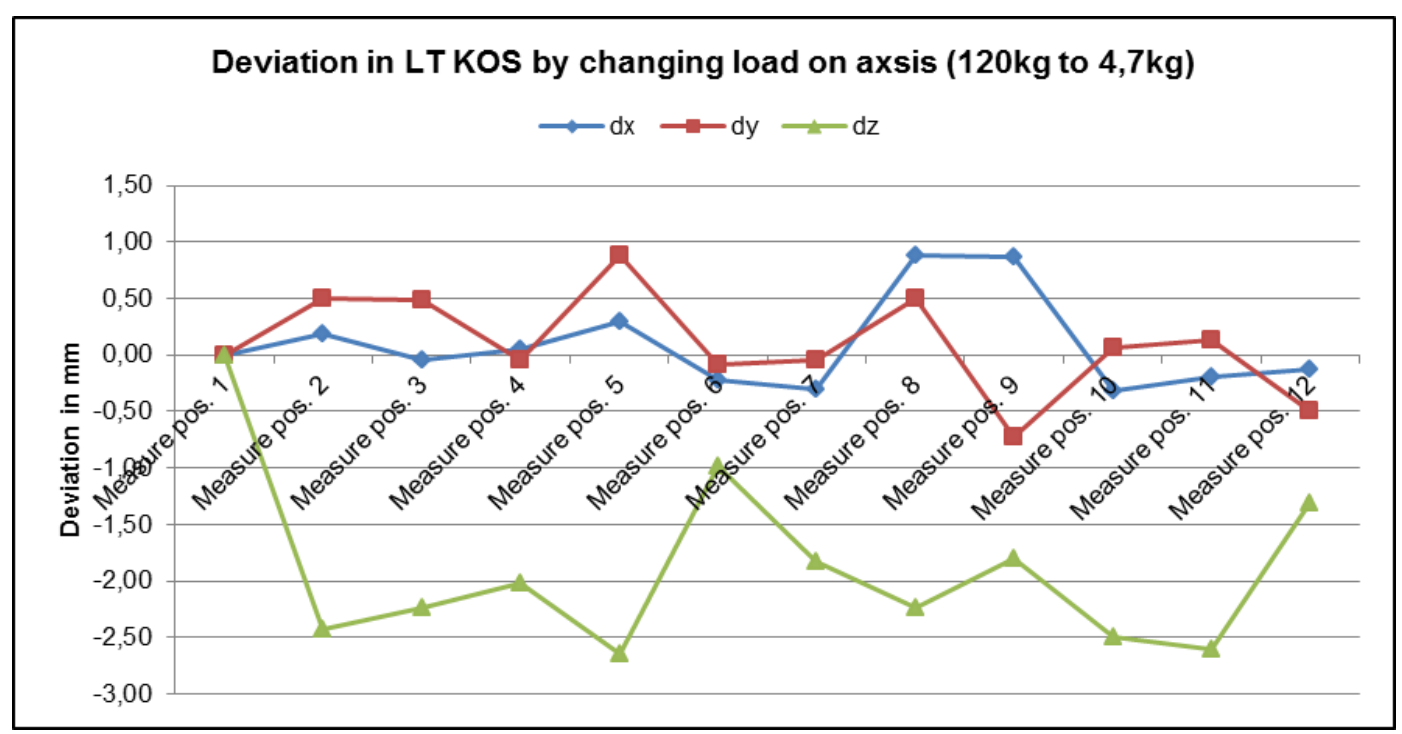

Figure 5: Positions deviation influenced by loading in IQZ robot cell

Another experiment in the TEZ robot cell with an external linear axis shows the positions deviation. It shows that deviation of axis values differs and that depends on robot position and orientation in the cell. The results are presented in figure 6 . 


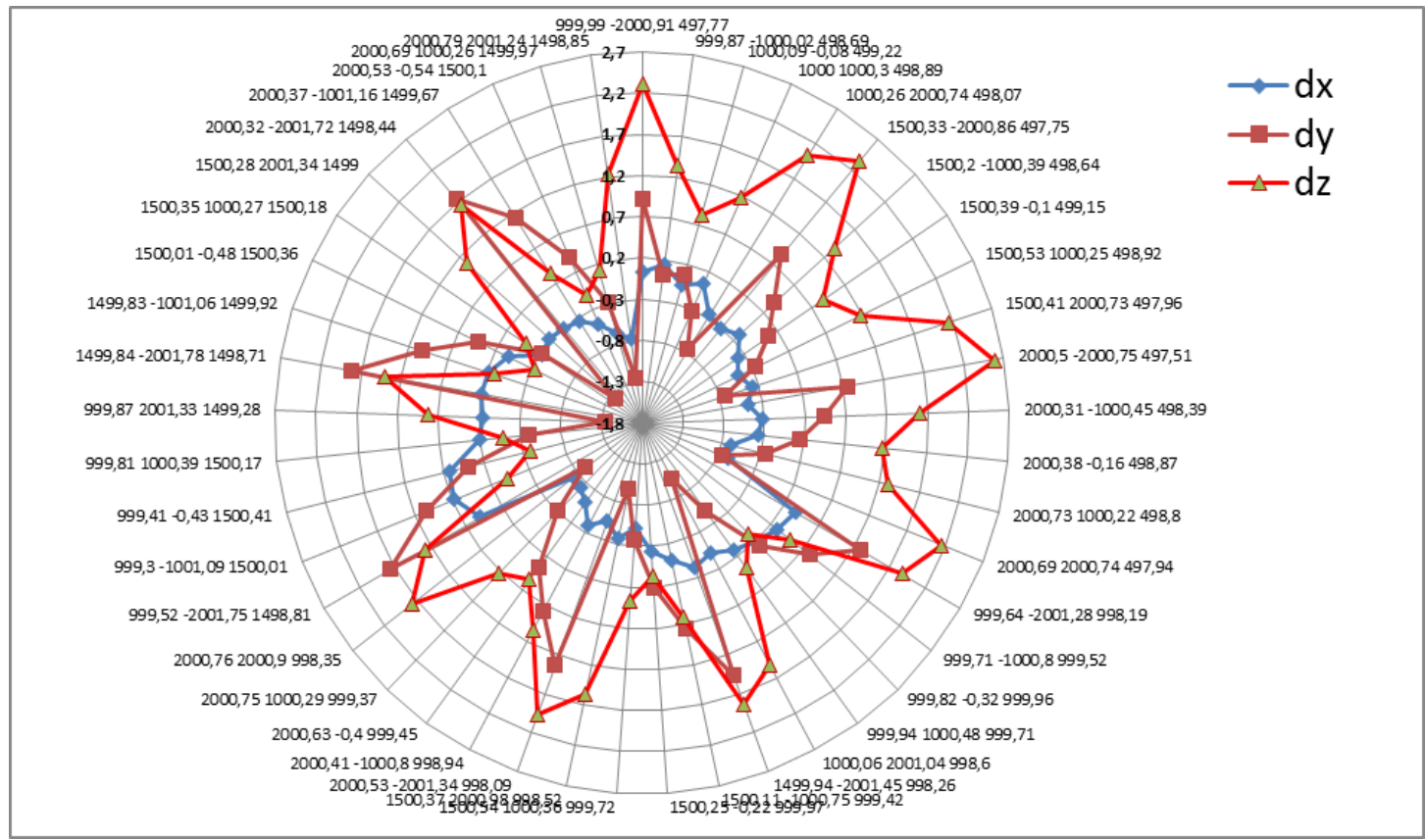

Figure 6: Positions deviation influenced by external linear axis in TEZ robot cell

A further experiment in IQZ robot cell demonstrates the temperature influence on robot position accuracy. It is not possible for technical reasons to completely avoid the influence of temperature on the kinematic chain as the joint gear groups of the kinematic chain under load generate heat, which leads to time-dependent changes of the same length and angle. By a thermo-symmetrical design, these effects cannot be completely excluded. In this experiment the robot has been moved continuously for approx. five hours within predefined random 12 positions. During this robot movement in every $30 \mathrm{~min}$ the positions are measured by laser tracer. The figure 7 shows that, the temperature of all axes (axis 1 to axis 6) has been along with time increased. This leads to a time-dependent position deviation of robot. Figure 8 represents the positions deviation in magnitude.

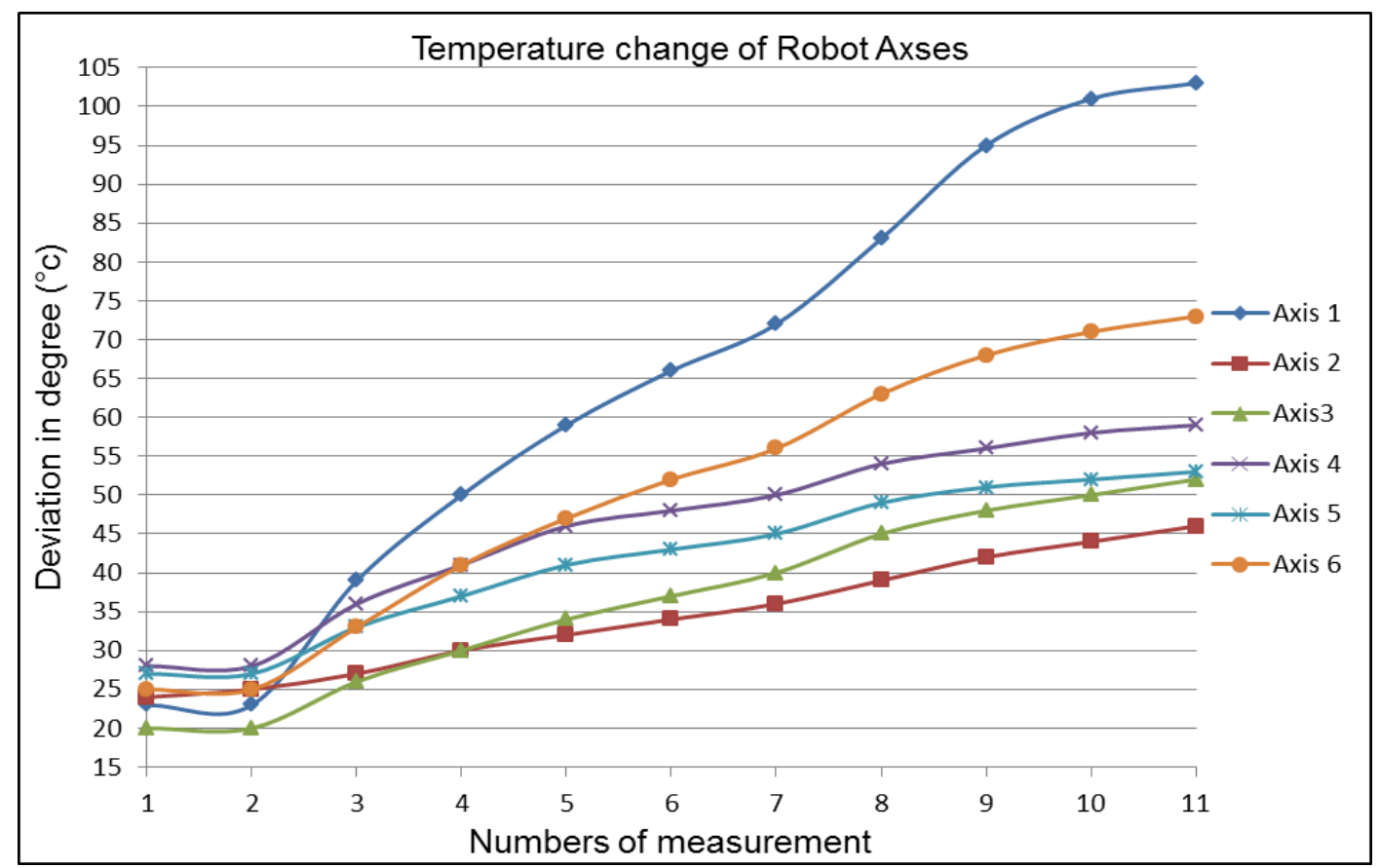

Figure 7: increased Axis temperature 


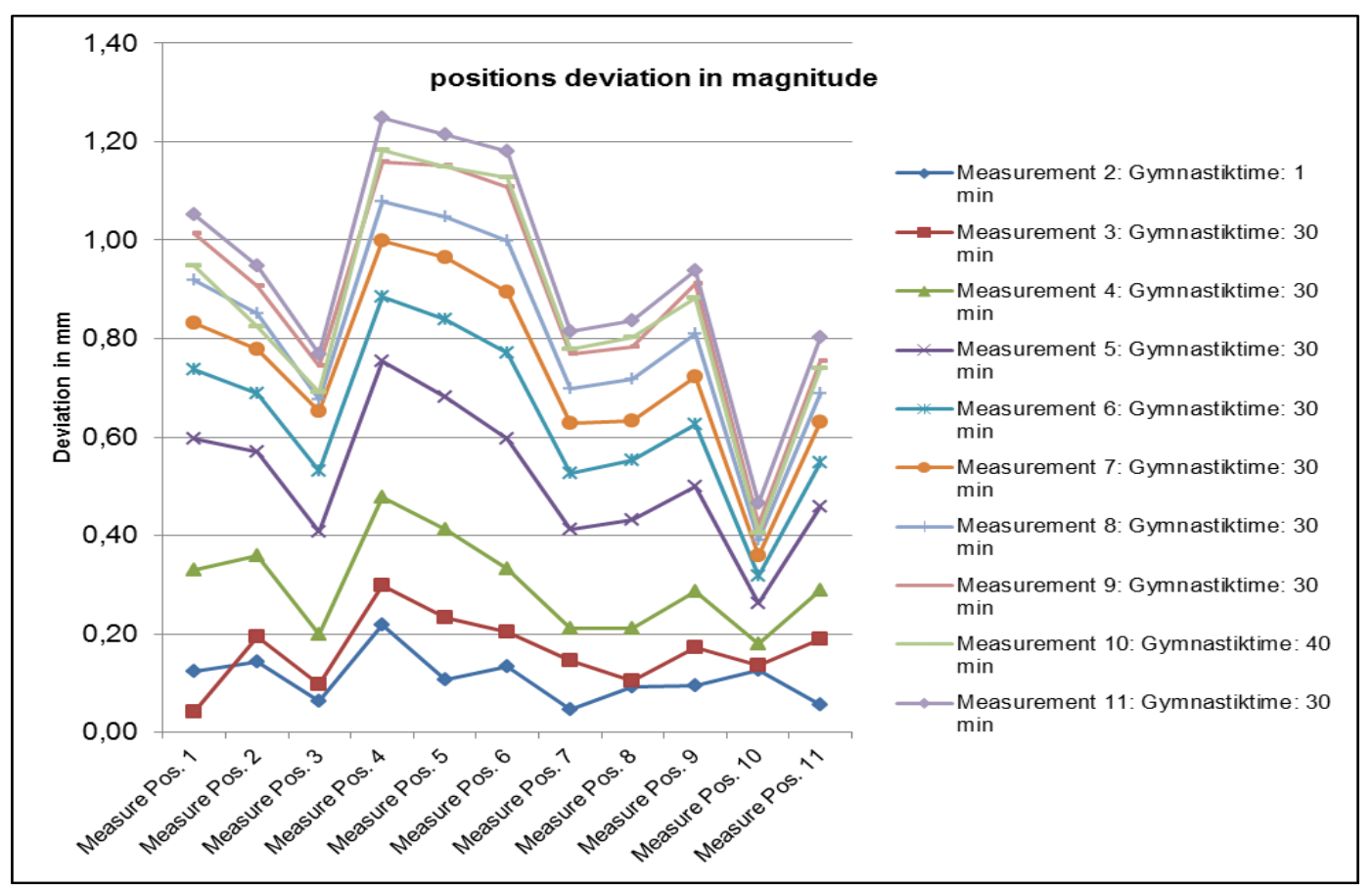

Figure 8: Position deviation due to temperature incensement

\subsection{Measuring System for accuracy improvement}

Many fields of investigation exist to increase the accuracy of industrial robots like: robot calibration, process development and control system. Robot calibration improves the accuracy of positioning by reducing the deviation between the commanded pose and the real one. Different techniques also exist to compensate these deviations. The sensor based method offers good position accuracy. However its implementation on an existing robot is difficult. Many problems could be encountered like the positioning of sensors on the robot structure and their integration in the control loop besides the extra cost due to the integration of these sensors. Figure 8 represents few of those existing sensor based measuring system to improve the robot accuracy.

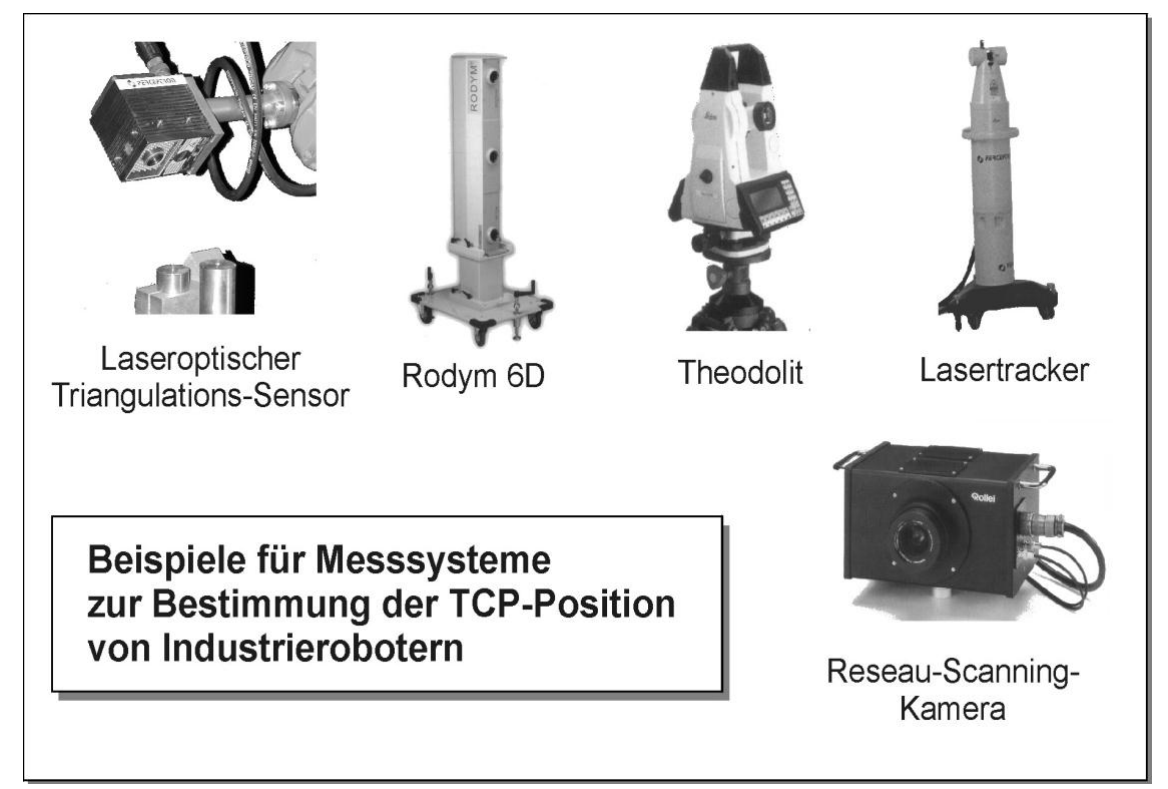

Figure 9: sensor based Measurement to improve the robot accuracy 


\section{Concept and approach}

One of the main technological barriers in the robotics industry has been the reduction of error between the tool frame and the goal frame. Advanced OLP software packages exist, in which the entire cell, the robot and all the machines or instruments in the workpiece are mapped graphically. The technique has limited value because it relies on accurate measurement of the positions of the associated equipment, and also relies on the positional accuracy of the robot which may or may not conform to what is programmed.

The present technique relates to a method for projecting and determination of the measurement field, in particular to the orientation of the measurement position in a larger component. This concept is based on absolute positioning methods, which estimate the robot position and orientation in the workpiece reference by detecting contactless marker projected by laser projector on the workpiece in the robot environment. Figure 10 represents the concept for measuring the position of robot guided workpiece.

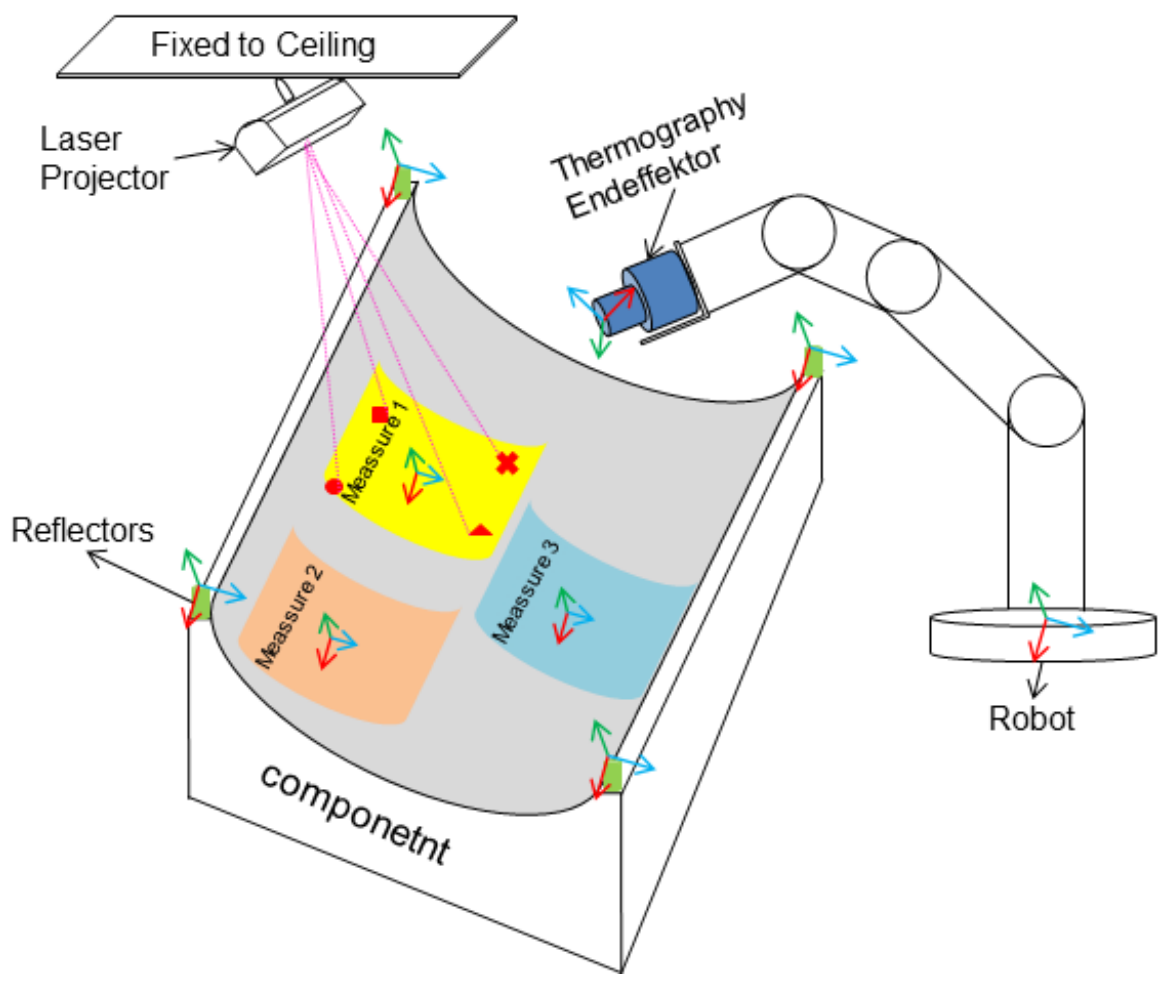

Figure 10: Concept for measuring the position of robot guided workpiece

In this concept first of all the component location to the laser projector has to be calibrated. The method of calibrating the location of a laser projector relative to a workpiece utilizes a series of randomly-placed reference points or so called reflectors, with the points being spaced by a known distance. For this purpose according to CAD Model 4 reflectors are attached to the corresponding point on the component and measured with the laser, the positions of the reflectors mounted on the component. After the workpiece calibration laser projector will project measurement field (pre calculated distribution of measurement field, due to lateral thermal wave diffusion of Lock-In Thermography) one by one. Measurement field size and number of measurements field position on the component is calculated and defined in the CAD depending on part geometry and component size. Based on pre calculated measurement field an offline program for robot target position relative to measurement field can be generated. For offline program tool center point is located on Thermography camera lense.

The measurement field projected by laser projector is consists of 4 distinctly different features (e.g.: square, circle, circle ring, cross). The individual position of each feature as well as relative position to each other feature on the component is known. These projected features on the component increases local temperature (as like hot spot). The thermography camera which is used for non-destructive-testing can detect these features and generates thermal image. In addition to that for the offline program these features are considered as reference or target position for camera field. Due to the modelling differences between ideal world (CAD, OLP, 100\% robot position accuracy etc.) and real world (Manufactured equipment, measuring accuracy and robot position inaccuracy) the robot actual position relative to target measurement 
field position has to be improved. Figure 11 represents a possible example of position deviation between robot target position and the robot actual position.

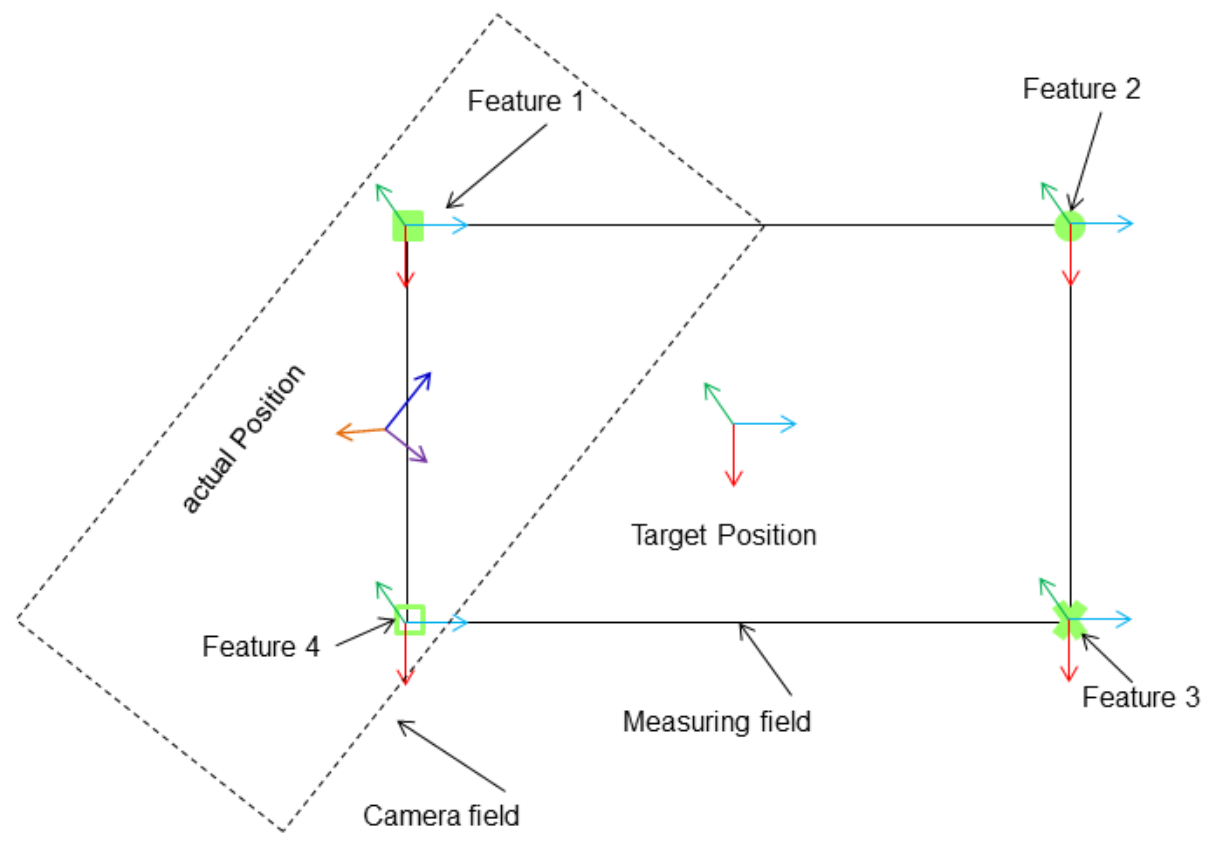

\section{Figure 11: Possible example of position deviation between robot target position and the robot actual position}

Under static condition, it is possible to calculate the differences between reference position and orientation of the thermography camera field and the target position (measurement field) where the features are projected on the component (measurement field).

DLR Augsburg is working on this concept and developing an appropriate algorithm to minimize the differences between the target position and actual position. However, since the measurements field through laser projector as contactless features on the component accurately projected and the thermography camera detects the features, can a iteration algorithms (in which the robot with the thermography camera will realign until the camera brings the features of measurement field to the given pixel position on the CCD chip) the robot position can be improved to the measurement field. The accuracy of the algorithm depends upon the features arrangements, the position of the robot in the robotic cell and on the laser projector based contactless feature resolution.

In addition to that, with the help of these contactless features and their exact position on the component, thermography measurement pictures can be stitched together to get one complete measurement picture of hole component. These will help to find out and mark out the detected damages easily.

\section{Conclusions}

Industrial robot with six degree of freedom has more flexibility than any other devices in automated factories. In this paper, a method that copes with the dynamic positioning problem has been presented. The method combines industry robot kinematics, laser projector and a NDT method as Thermography. The new concept consists firstly in the development of the laser device for projecting the measurement field, secondly in the development of an algorithm to improve the robot position at the measurement pitch. The advantages of the system are:

- With this method, the inaccuracy of the robot can be neglected because the robot position to the relative measurement field is improved. Thus, no "high accuracy" is robot needs.

- In this case, no expensive external sensor based system is needed.

- No additional camera systems for the detection of the measurement field are required. But only with the thermography camera, which is as well as applied to the component inspection, is used for detection. 
http://dx.doi.org/10.21611/qirt.2014.023

- The system can be applied for each complex component (not component specific).

- The condition of the system will be so very simple that no training is required.

\section{REFERENCES}

[1] T Schmidt, S Dutta. "Automation in Production Integrated NDT Using Thermography", NDT in Aerospace, Augsburg 2012

[2] T Schmidt, S Dutta. "CFRP manufacturing process chain observation by means of automated thermography" 5th International Symposium on NDT in Aerospace, 13-15th November 2013, Singapore 